\title{
Theta and Gamma Oscillations during Encoding Predict Subsequent Recall
}

\author{
Per B. Sederberg, ${ }^{1}$ Michael J. Kahana, ${ }^{1,3}$ Marc W. Howard, ${ }^{2}$ Elizabeth J. Donner, ${ }^{4}$ and Joseph R. Madsen ${ }^{3,5}$ \\ ${ }^{1}$ Volen Center for Complex Systems, Brandeis University, Waltham, Massachusetts 02454, ${ }^{2}$ Department of Psychology, Syracuse University, Syracuse, New \\ York 13244, Departments of ${ }^{3}$ Neurosurgery and ${ }^{4}$ Neurology, Children's Hospital Boston, Boston, Massachusetts 02115, and ${ }^{5}$ Department of Surgery, \\ Harvard Medical School, Boston, Massachusetts 02115
}

\begin{abstract}
Electrophysiological and hemodynamic measures of human brain activity have been shown to distinguish between episodes of encoding items that are later recalled versus those that are not recalled (Paller and Wagner, 2002). Using intracranial recordings from 793 widespread cortical and subcortical sites in 10 epileptic patients undergoing invasive monitoring, we compared oscillatory power at frequencies ranging from 2 to $64 \mathrm{~Hz}$ as participants studied lists of common nouns. Significant increases in oscillatory power during encoding predicted subsequent recall, with this effect predominantly in the $4-8 \mathrm{~Hz}$ (theta) and $28-64 \mathrm{~Hz}$ (gamma) frequency bands. Sites exhibiting increased theta activity during successful encoding were clustered in right temporal and frontal cortex, whereas those exhibiting increased gamma activity appeared bilaterally at widespread cortical locations. These findings implicate theta and gamma oscillatory activity, across a widespread network of cortical regions, in the formation of new episodic memories.
\end{abstract}

Key words: theta; gamma; iEEG; free recall; subsequent memory effect; epilepsy

\section{Introduction}

Physiological activity during encoding must participate in transforming experience into a lasting trace that can later be recalled and recognized. Subsequent memory paradigms, in which online measures of neural activity as people encode items are segregated by the subsequent ability to recall or recognize those items, have helped to characterize the activity of different brain regions during memory formation (Paller and Wagner, 2002).

In subjects faced with remembering a list of items, both evoked potentials and hemodynamic activity measured during encoding exhibit reliable differences between subsequently recalled and not recalled items (Rugg and Allan, 2000; Paller and Wagner, 2002). This effect has been termed the subsequent memory effect (SME). Recording intracranially in the medial temporal lobe (MTL), Fernandez et al. (1999) have shown that the differences that predict recall appear both as a negative deflection in the rhinal cortex event-related potential (ERP) $\sim 400 \mathrm{msec}$ after stimulus exposure and as a positive hippocampal ERP beginning $\sim 800 \mathrm{msec}$ after stimulus onset. This finding provides important constraints on the MTL generator of the SME observed at the parietal scalp (Rugg and Allan, 2000) and perhaps also the MTL SME observed using functional magnetic resonance imaging

Received June 16, 2003; revised Sept. 22, 2003; accepted 0ct. 6, 2003.

This work was supported by National Institutes of Health Research Grants MH55687 and MH61975 (M.J.K.). This work was made possible by the cooperation of colleagues in the Epilepsy Program at Children's Hospital Boston, including P. M. Black. We thank Kelly Addis, Jeremy Caplan, Arne Ekstrom, Sri Raghavachari, and Dan Rizzuto for helpful discussions and feedback on this manuscript. We are grateful to Emily Dolan, David Seelig, and Michele Tully for data collection and processing.

Correspondence should be addressed to Dr. Michael Kahana, Volen Center for Complex Systems, Brandeis University, Waltham, MA 02454. E-mail: kahana@brandeis.edu.

Copyright $\odot 2003$ Society for Neuroscience $\quad$ 0270-6474/03/2310809-06\$15.00/0
(fMRI) (Brewer et al., 1998; Wagner et al., 1998; Kirchhoff et al., 2000; Strange et al., 2002).

Recently, researchers have begun to examine the role of oscillations, inherent in both scalp and intracranial EEG (iEEG) potentials, in memory function. In iEEG recordings from MTL, Fell et al. (2001) observed that increased gamma synchrony between the rhinal cortex and the hippocampus, but decreased gamma power, predicted subsequent recall of words. The role of oscillations in cognitive processing is not confined to the gamma frequency band. In particular, oscillations in the $4-8 \mathrm{~Hz}$ theta band have been found to increase with cognitive demands during spatial learning (Kahana et al., 1999; Caplan et al., 2001), spatial navigation (de Araujo et al., 2002; Caplan et al., 2003), verbal and spatial working memory (Gevins et al., 1997; Krause et al., 2000; Tesche and Karhu, 2000; Raghavachari et al., 2001; Rizzuto et al., 2003), and item recognition (Klimesch et al., 1997; Klimesch, 1999).

Oscillations at lower frequencies, including theta, have also been linked to episodic recall. Klimesch et al. (1996) found significant increases in scalp-recorded theta power during episodic encoding of words that were subsequently recalled. Similarly, Weiss and Rappelsberger (2000) found increased oscillatory coherence between frontal and posterior scalp electrode sites, as well as significant increases in $1-4 \mathrm{~Hz}$ power, for both visually and auditorally presented, subsequently recalled nouns. Fell et al. (2003) also found increased theta coherence between intracranially recorded electrodes during encoding of successfully recalled words but did not observe any significant change in theta power.

Extensive animal work also points to the functional importance of theta oscillations. Long-term potentiation depends on the phase of hippocampal theta activity, with enhanced synaptic plasticity at the peak of the theta oscillation and decreased plas- 
Table 1. Participant information

\begin{tabular}{|c|c|c|c|c|c|c|}
\hline Participant & Age & Gender & Resection & $\begin{array}{l}\text { Number of } \\
\text { electrodes }\end{array}$ & $\begin{array}{l}\text { Nunber } \\
\text { excluded }\end{array}$ & $\begin{array}{l}\text { Percentage } \\
\text { recalled }\end{array}$ \\
\hline 1 & 13 & $\mathrm{~F}$ & L amygdala/anterior hippocampus & 64 & 10 & 25.42 \\
\hline 2 & 12 & $\mathrm{~F}$ & R motor-sensory transection & 104 & 12 & 13.33 \\
\hline 3 & 15 & M & R anterior temporal & 128 & 36 & 15.00 \\
\hline 4 & 17 & M & L inferior/medial temporal & 64 & 16 & 19.89 \\
\hline 5 & 15 & M & L anterior frontal & 123 & 11 & 28.00 \\
\hline 6 & 11 & M & R angular gyrus & 104 & 17 & 18.17 \\
\hline 7 & 14 & $\mathrm{~F}$ & L temporal & 72 & 7 & 34.67 \\
\hline 8 & 8 & $\mathrm{~F}$ & R temporal & 88 & 7 & 24.64 \\
\hline 9 & 17 & M & L temporal & 90 & 19 & 10.71 \\
\hline 10 & 17 & $M$ & None & 124 & 33 & 11.37 \\
\hline
\end{tabular}

$F$, Female; $M$, male; $L$, left; $R$, right.

ticity at the trough (Pavlides et al., 1988; Huerta and Lisman, 1993; Hölscher et al., 1997). During a working memory task, hippocampal theta appears phase locked to stimuli (Givens, 1996), providing a possible mechanism for sensory encoding (Hasselmo et al., 2002). Furthermore, blocking theta oscillations by lesioning the medial septum causes severe memory impairments (Givens and Olton, 1990; Leutgeb and Mizumori, 1999).

These findings concerning the general involvement of theta and gamma oscillations in both animal and human memory function lead us to ask whether cortical brain oscillations play a direct role in the successful encoding of information into longterm memory. Specifically, we investigated whether changes in oscillatory activity during encoding predict successful recall. We examined oscillatory power across a wide range of frequencies as participants with implanted electrodes performed an episodic memory task. We asked subjects to study a list of individually presented words, and then, after a filled delay period, subjects attempted to recall as many words as possible, in any order. This type of free-recall task has been shown to be especially well suited for studying the SME (Rugg and Allan, 2000).

\section{Materials and Methods}

Subjects. We tested 10 subjects (ages 8-17 years; four female) who had been surgically implanted with subdural electrodes on the cortical surface. The clinical team determined the placement of these electrodes so as to best localize epileptogenic regions. We recorded from a total of 961 electrodes; 168 of these were (1) in the epileptic focus, (2) overlying regions of radiographically evident structural brain damage, or (3) exhibited epileptiform EEG (i.e., spikes and/or sharp waves as determined by the clinical team). We restricted our analyses to the remaining 793 electrodes. Table 1 reports each participant's age, gender, region of resection, total number of electrodes, number of excluded electrodes, and average recall performance. All participants were tested for their reading ability, and none had difficulty reading the nouns used as stimuli in our study. The variability in their recall performance was reasonable considering their varied ages and the fact that they were tested at their hospital bedside. All subjects had normal range personality and intelligence. Our research protocol was approved by the institutional review board at Children's Hospital Boston, and informed consent was obtained from the subjects and their guardians.

Procedure. Subjects studied lists of words for a delayed free-recall task. Lists were composed of 20 common nouns, chosen at random and without replacement from a pool of high-frequency nouns (mean frequency of 49.0; http://fechner.ccs.brandeis.edu/wordpools.php). Over the course of one to four sessions, subjects received 12-60 study-test lists (the number of trials obtained depended on the patient's interest and availability for testing). A computer controlled stimulus presentation and recorded subjects' responses. At the start of each trial, a plus sign appeared at the center of the screen to alert subjects to the upcoming word presentation and to encourage them to fixate on the center of the screen. The plus sign appeared for $1600 \mathrm{msec}$, followed by a $800-1200$ msec blank interstimulus interval (ISI). The computer then displayed each list item in capital letters for $1.6 \mathrm{sec}$, followed by an $800-1200 \mathrm{msec}$ blank ISI. This temporal jitter served to decorrelate the physiological responses from successive word presentations. To ensure that each word was attended to, we asked that subjects read each word aloud as soon as it appeared. All subjects readily complied with these instructions.

Immediately after each list presentation, subjects were given a series of simple arithmetic problems. This end-of-list distractor task served to reduce the large advantage accorded to end-of-list items during recall (Howard and Kahana, 1999). Each problem took the form of $A+B+$ $C=$ ??, where $A, B$, and $C$ were randomly chosen positive integers from the set one through nine. Subjects were asked to respond vocally as soon as they knew the answer. The experimenter typed their answer into the keyboard. Each entry evoked one of two tones from the computer speaker, depending on whether it was correct or incorrect. After subjects solved arithmetic problems for $16 \mathrm{sec}$, the subject's last arithmetic problem was immediately followed by a row of asterisks accompanied by a 300 msec tone signaling the start of the recall period. Subjects were given 46 sec to recall list items in any order (standard free-recall instructions). After each session, vocal responses, digitally recorded during the trial, were scored for analysis.

$i E E G$ recordings. The iEEG signal was recorded from platinum electrodes ( $3 \mathrm{~mm}$ diameter) with an inter-electrode spacing of $1 \mathrm{~cm}$. The signal was amplified, sampled at $256 \mathrm{~Hz}$, and bandpass filtered between 0.3 and $70 \mathrm{~Hz}$ (apparatus from Bio-Logic Systems, Mundelein, IL). Data were subsequently notch filtered using Sleppian sequences at 60 and 44 $\mathrm{Hz}$ to eliminate electrical line and equipment noise.

To synchronize the electrophysiological recordings with behavioral events, the experimental computer sent pulses through the parallel port via an optical isolator into an unused recording channel. The time stamps associated with these pulses enabled the alignment of the experimental clock of the computer with the iEEG clock to a precision well under the sampling interval of the iEEG recording $(<4 \mathrm{msec})$. For all subjects, the locations of the electrodes were determined using coregistered postoperative computed tomographies and preoperative MRIs by an indirect stereotactic technique and converted into Talairach coordinates.

Oscillatory power analyses. As in previous iEEG work (Kahana et al., 1999; Caplan et al., 2001, 2003; Howard et al., 2003; Rizzuto et al., 2003), we used the Morlet wavelet transform (with wave number $=6$ ) to compute the spectral power as a function of time for all of our iEEG signals. Frequencies were sampled logarithmically at 41 intervals between 2 and $64 \mathrm{~Hz}$. Because the wavelet scales with frequency, it allows for comparisons between time-varying high-frequency and low-frequency signals on an equal footing. This is especially important for EEG signals, which are generally nonstationary and have time-varying phase components (for additional details, see Caplan et al., 2001). Additionally, wavelets have Gaussian impulse functions that provide a true time-frequency distribution but avoid edge effects and issues of windowing that are inherent in fast Fourier transform methods.

A Wilcoxon rank sum test was then used to compare items that were later recalled with items that were not recalled using the mean of wavelet power during the encoding period ( $2 \mathrm{sec}$ interval after presentation on- 
set). This comparison was made separately for each electrode and at each frequency.

We generated an unbiased empirical estimate of the type I error rate using a bootstrap procedure (Efron, 1979), a method often used to handle correlations among statistical comparisons of electrophysiological measures (Lachaux et al., 1999; De Gennaro et al., 2000; Graimann et al., 2002; Howard et al., 2003; Rizzuto et al., 2003). The bootstrap procedure first entails generating 1000 random samples of the experimental data by randomly swapping items designated as recalled and not recalled for each subject. We used the same randomization across electrodes to control for between-electrode correlations. Next we performed the Wilcoxon rank sum test on the 1000 random shuffles of data, generating a distribution that allowed us to determine the Wilcoxon rank sum statistic, $W_{s}$, threshold that would set the type I error rate at $0.5 \%$ of a subject's electrodes per frequency. Although the same basic pattern of results can be seen for a range of type I error rates, using a more liberal threshold (e.g., 1.0\% of a subject's electrodes) would make it difficult to interpret the brain maps (too many false positives). Similarly, using a more conservative threshold (e.g., $0.1 \%$ of a subject's electrodes) would have only picked out the most significant sites, making it impossible to compute aggregate time courses for the effects.

If $W_{s}$ exceeded the empirically determined threshold, that particular frequency and electrode was deemed to exhibit a significant oscillatory SME. A positive SME at a given frequency for a given electrode indicates significantly greater power during the encoding of words that are later recalled; a negative SME indicates significantly greater power during encoding of words that are later not recalled.

To illustrate the time course of the oscillatory SMEs, we calculated time-frequency significance spectrograms of the difference between recalled and not recalled power. We first averaged power across significant electrodes within each subject. We then calculated a $p$ value for the difference between recalled and not recalled power across subjects for each frequency from 2 to $64 \mathrm{~Hz}$ and each point in time from 0 to $2 \mathrm{sec}$ after the onset of each item presentation.

\section{Results}

Out of 793 recording sites, 140 distinct sites exhibited positive oscillatory SMEs. This number of sites far exceeded the type I rate $(p<0.0001)$. Figure 1, $a$ and $b$, illustrates two representative sites that exhibited positive oscillatory SMEs. Figure $1 a$, which illustrates data recorded from an electrode in the inferior frontaltemporal region of subject 3 , had a peak frequency in the $4-8 \mathrm{~Hz}$ theta frequency band. Figure $1 b$ plots data recorded from an electrode in the left temporal-occipital region of subject 4 with a peak frequency in the $28-64 \mathrm{~Hz}$ gamma band.

Figure $1 c$, which plots the number of sites exhibiting positive oscillatory SMEs at each frequency, shows that the dominant frequencies for this effect are in the theta and gamma frequency bands. Figure $1 d$ illustrates the locations of the 51 distinct electrodes exhibiting a positive oscillatory SME in the theta band; Figure 1e illustrates the locations of the 45 distinct electrodes exhibiting a positive oscillatory SME in the gamma band. Electrodes exhibiting increases in theta oscillations that predicted successful recall clustered in right temporal and frontal areas (34 of 51 electrodes) compared with the left temporal and frontal areas ( 3 of 51 electrodes). This lateralization effect was statistically significant $(p<0.0001)$. Sites at which increased gamma oscillations positively correlated with subsequent recall were equally distributed across left and right brain regions ( 22 in the right temporal and frontal areas; 18 in the left temporal and frontal areas; NS).

We also found electrodes exhibiting significant negative SMEs, with decreased power during the encoding of words that were later recalled. Of 793 recording sites, 131 distinct sites exhibited negative oscillatory SMEs (this number exceeded the type I rate; $p<0.0001)$. Figure $2 a$, which illustrates data recorded a.

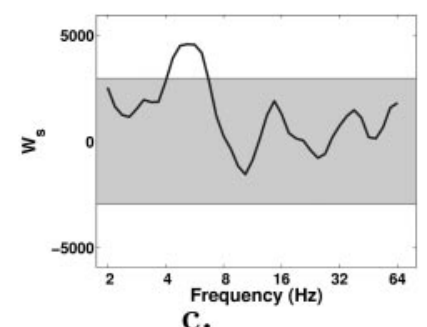

b.

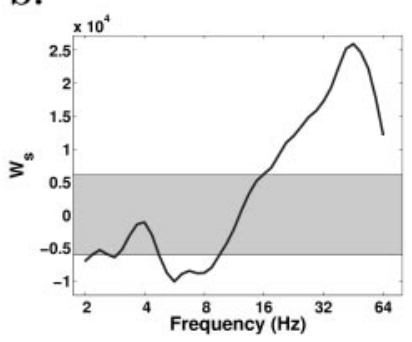

C.

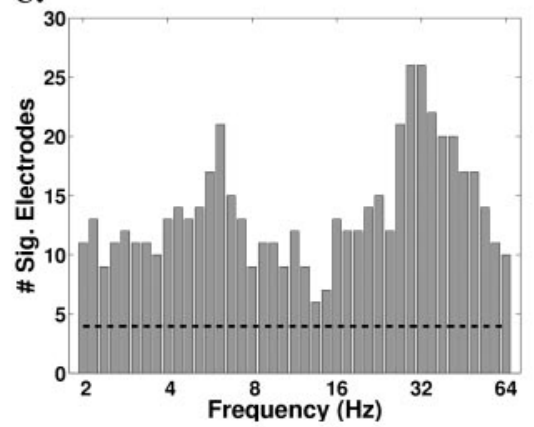

d.

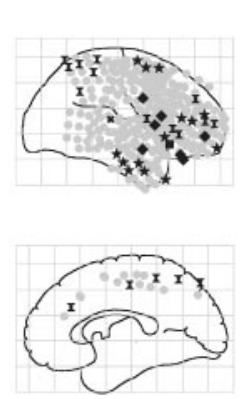

$4-8 \mathrm{~Hz}$
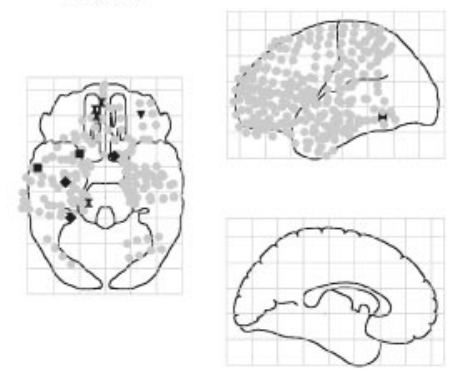

e.

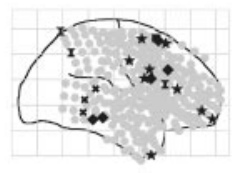

$28-64 \mathrm{~Hz}$
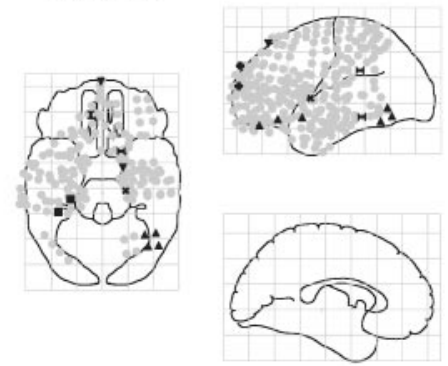

Figure 1. Increased oscillatory power during encoding predicts subsequent recall. $a$ plots the Wilcoxon rank sum statistic $\left(W_{s}\right)$ for the difference in power between recalled and not recalled words across frequencies from 2 to $64 \mathrm{~Hz}$ for recordings taken from an electrode in the inferiortemporal region of subject 3 [Talairach coordinates (left-right, anterior-posterior, inferiorsuperior $)=(-7.1,8.3,-18.3)]$. At this site, power in the $4-8 \mathrm{~Hz}$ theta band was higher during the encoding of subsequently recalled words. (The filled gray region represents the $95 \%$ confidence interval.) $b$ shows data taken from an electrode in the left temporal-occipital region of subject 4 [Talairach coordinates (left-right, anterior-posterior, inferior-superior) = $(-58.3,-57.9,-3.5)]$. At this site, power in the $28-64 \mathrm{~Hz}$ gamma band was higher during the encoding of subsequently recalled words. c shows the number of electrodes exhibiting significantly greater oscillatory power during successful encoding across frequencies from 2 to $64 \mathrm{~Hz}$. The dashed black line indicates the type I error rate for this analysis (see Materials and Methods). $d$ illustrates the locations of electrodes exhibiting significant power increases in the 4-8 Hz theta band during successful encoding (black symbols; each symbol represents electrodes from a single subject) and those that did not (gray circles). Electrode locations are illustrated on five views of a standard brain. Top left, Right lateral view. Top right, Left lateral view. Center, Inferior view. Bottom left, Left sagittal interhemispheric view. Bottom right, Right sagittal interhemispheric view. e shows the analogous effect for the $28-64 \mathrm{~Hz}$ gamma band. 


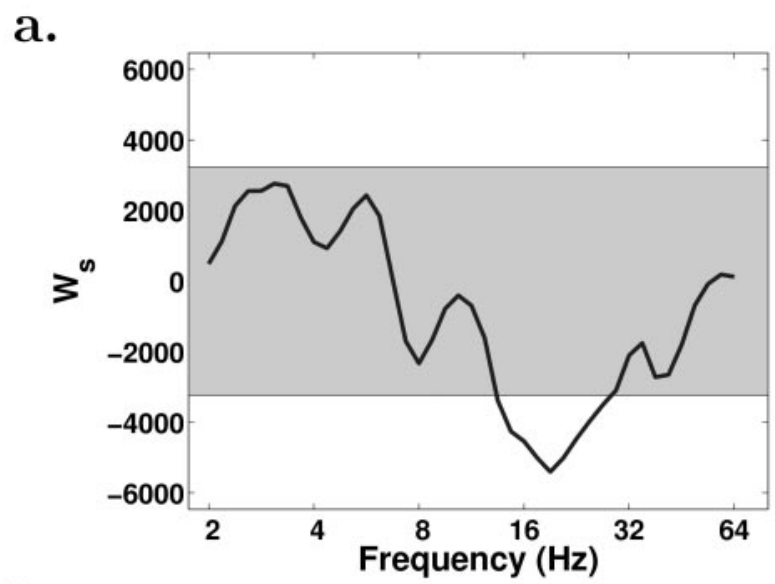

b.

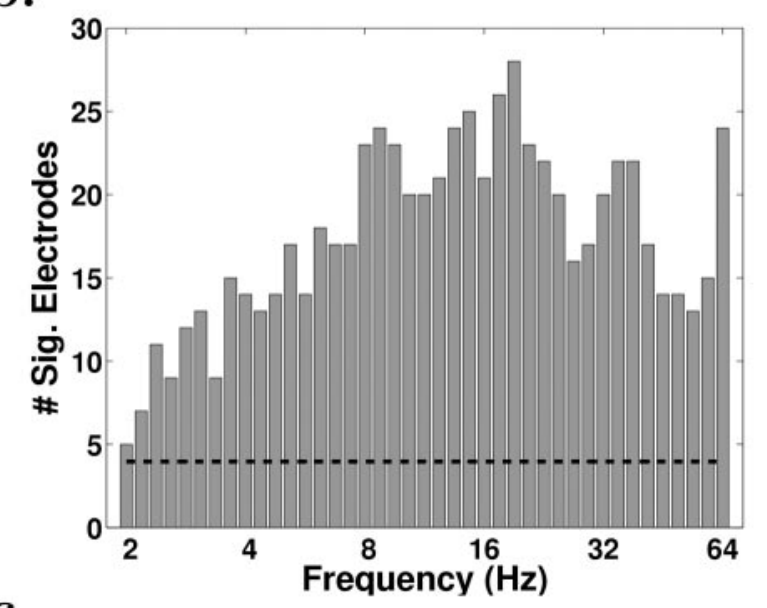

c.

\section{$9-28 \mathrm{~Hz}$}

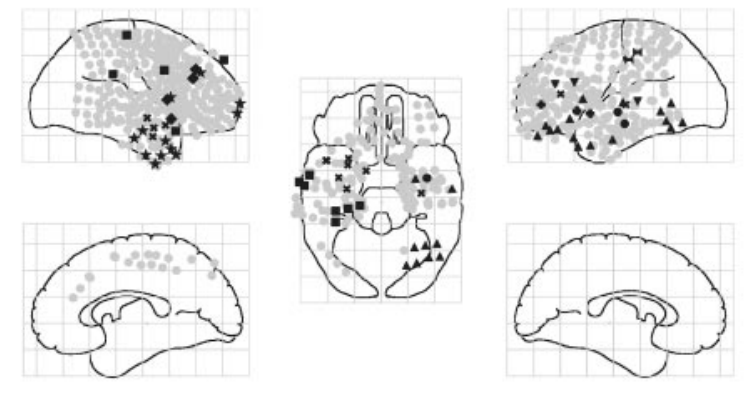

Figure 2. Decreased oscillatory power during encoding predicts subsequent recall. $a$ plots the Wilcoxon rank sum statistic $\left(W_{s}\right)$ for the difference in power between recalled and not recalled words across frequencies from 2 to $64 \mathrm{~Hz}$ for recordings taken from an electrode in the left inferior-temporal region of subject 8 [Talairach coordinates (left-right, anterior-posterior, inferior-superior $)=(-30.0,-17.0,-36.3)]$. At this site, power in the $9-28 \mathrm{~Hz}$ alpha/beta band was lower during the encoding of subsequently recalled words. (The filled gray region represents the $95 \%$ confidence interval.) $b$ shows the number of electrodes exhibiting significantly lower oscillatory power during successful encoding across frequencies from 2 to $64 \mathrm{~Hz}$. The dashed black line indicates the type l error rate for this analysis (see Materials and Methods). c illustrates the locations of electrodes exhibiting significant power decreases in the $9-28 \mathrm{~Hz}$ alpha/beta band during successful encoding (black symbols; each symbol represents electrodes from a single subject) and those that did not (gray circles). The topographical maps follow the same format seen in Figure 1.

from an electrode in the left inferior-temporal region of subject 8 , had a peak frequency in the $9-28 \mathrm{~Hz}$ alpha/beta frequency band.

Figure $2 b$, which plots the number of sites exhibiting negative oscillatory SMEs at each frequency, shows that the dominant frequencies for this effect are in the alpha $(9-12 \mathrm{~Hz})$ and beta $(13-28 \mathrm{~Hz})$ frequency bands. Figure $2 c$ illustrates the locations of the 79 distinct electrodes exhibiting a negative oscillatory SME in the 9-28 Hz band. Electrodes exhibiting decreases in alpha/beta oscillations that predicted successful recall clustered in both the right and left temporal and frontal areas but were equally distributed across the left and right hemispheres (36 right frontal and temporal; 38 left frontal and temporal; NS).

The foregoing analyses determined oscillatory SMEs based on the $2 \mathrm{sec}$ period after each stimulus presentation. We next examined the time course of these effects by computing the average time-frequency spectrogram for the electrodes exhibiting positive theta, positive gamma, and negative alpha/beta oscillatory SMEs. As shown in Figure $3 a$, the positive theta SME was highly significant $\left(p<10^{-8}\right)$ in the $600-1300$ msec interval after stimulus onset. For the gamma SME sites (Fig. $3 b$ ), the average timefrequency spectrogram exhibits a similar time course, with significant differences between recalled and not recalled items beginning at $700 \mathrm{msec}$ after word onset. This analysis indicates that the positive oscillatory SMEs occur relatively late in the encoding of a to-be-remembered word, suggesting a role for theta and gamma oscillations in associative or rehearsal processes.

The negative SME sites in the alpha/beta frequency range (Fig. $3 c$ ) only met our significance threshold during a short interval $\sim 1500$ msec after stimulus onset. Some of these negative SME sites simultaneously contributed to the positive SME at lower frequencies. Because these sites were distributed widely throughout cortex and across frequency bands, it is likely that their timing was not as consistent as that of the positive SME sites. This might have contributed to their weak showing in the time-frequency spectrogram.

\section{Discussion}

This study explored the role of cortical brain oscillations in memory formation. Specifically, we tested whether changes in oscillatory activity during the encoding of an item can predict its subsequent recall. In recording from 793 sites across 10 subjects, we found that theta $(4-8 \mathrm{~Hz})$ activity during encoding of successfully recalled words significantly increased at 51 sites, predominantly in right temporal and frontal cortex. Increases in gamma $(28-64 \mathrm{~Hz})$ activity significantly predicted subsequent recall at 45 widely distributed cortical sites. Some sites also showed decreases in oscillations during encoding of successfully recalled items, an effect that was broadband and did not localize to any particular brain structure.

Our finding that theta oscillations in human cortex increase during successful encoding is reminiscent of studies showing that, in rats, hippocampal theta oscillations gate synaptic plasticity both in vivo and in vitro (Pavlides et al., 1988; Huerta and Lisman, 1993; Hölscher et al., 1997). The increased theta activation localizing to the temporal and frontal lobes in humans may signify the recruitment of those regions during successful memory encoding.

Whereas we report that increases in cortical theta and gamma power predict successful memory encoding, Fell et al. (2001, 2003) found no changes in hippocampal theta power and decreases in hippocampal gamma power during encoding of subsequently recalled words. Whereas Fell et al. recorded from depth electrodes in hippocampal and parahippocampal regions, we recorded from electrode grids placed subdurally on the cortical surface. It is possible that theta oscillations recorded in small regions throughout the cortex play a different role and are gen- 


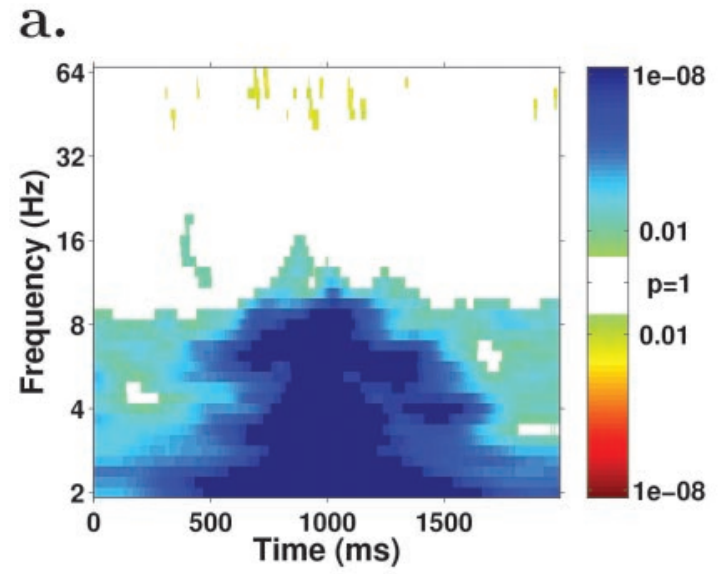

b.

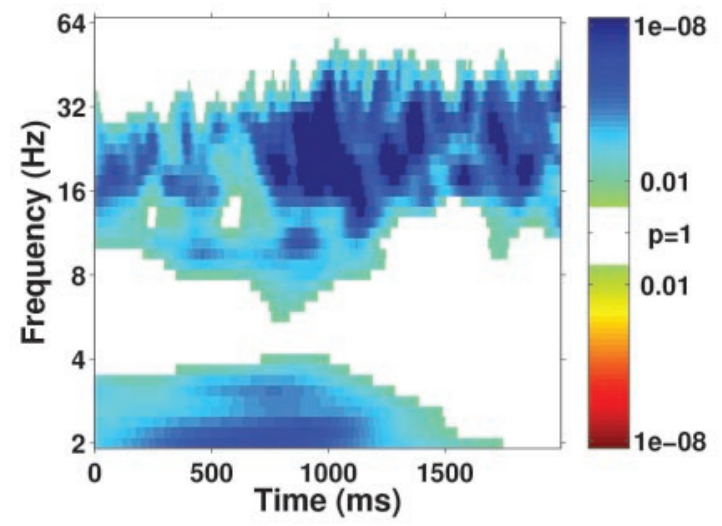

c.

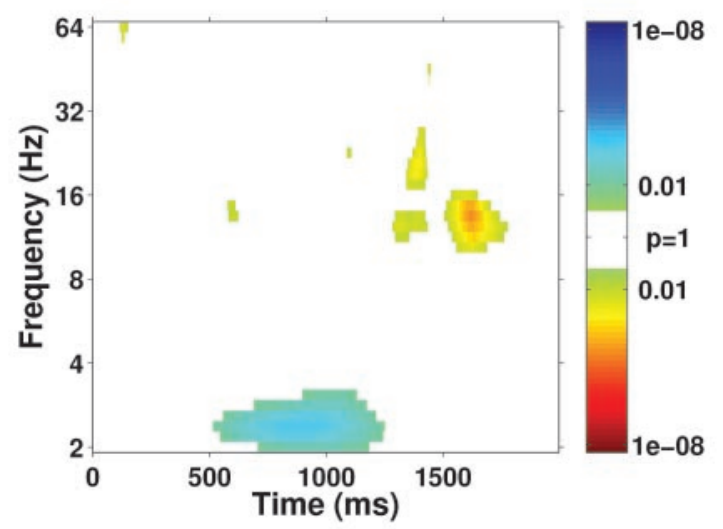

Figure 3. Time courses of oscillatory SMEs. Averaged across electrodes that exhibited a significant positive SME in the $4-8 \mathrm{~Hz}$ theta frequency band (see Materials and Methods), the shaded blue region in a illustrates times $(0-2 \mathrm{sec})$ and frequencies $(2-64 \mathrm{~Hz})$ when power was significantly greater during the encoding of subsequently recalled words. $b$ illustrates the average time course for electrodes exhibiting a significant positive oscillatory SME in the $28-64 \mathrm{~Hz}$ gamma frequency band. c plots the average time course for electrodes exhibiting a significant negative oscillatory SME in the alpha and beta bands. The color bar to the right of each panel plots the $p$ values for the difference between recalled and not recalled power (blue shades indicate significant increases in oscillations predicting recall, and red shades indicate significant decreases).

erated distinctly from the theta oscillations found in the hippocampus (Kahana et al., 2001).

Our finding of increased cortical gamma activity during successful encoding is consistent with the hypothesized role of gamma in top-down attentional processing. For example, gamma power at the scalp has been found to increase when subjects selectively attend to target stimuli (Tiitinen et al., 1993) and during the processing of rare, compared with common, targets (Debener et al., 2003). Tallon-Baudry et al. (1998) found that gamma power increased during the retention interval of a delayed match-to-sample task, interpreting the increase as related to rehearsal processes in working memory. Gamma power has also been examined intracranially and found to correlate with successful recognition of ambiguous objects (Rodriguez et al., 1999) and to increase with memory load during a working memory task (Howard et al., 2003). Thus, increased gamma power may be seen as reflecting increased allocation of attentional resources, which would in turn lead to better memory performance.

Lisman and Idiart (1995) and Fell et al. (2003) both discuss a possible interaction between theta and gamma oscillations underlying memory function. Specifically, Lisman and colleagues present a biophysical model of working memory in which simultaneously present theta and gamma oscillations allow for the segmentation of $7 \pm 2$ distinct patterns of neural activity (representing memories) that are repeated in each theta cycle (Lisman and Idiart, 1995; Jensen and Lisman, 1998). Our cortical results, however, show a very small positive correlation between electrodes exhibiting significant positive theta and gamma SMEs (discrete correlation Yule's $Q=0.23 \pm 0.25$; NS). This minimal degree of overlap between sites exhibiting significant theta and gamma SMEs indicates that different brain regions underlie these two physiological correlates of successful memory formation.

Our finding of numerous widespread sites exhibiting significant negative SMEs in the $9-28 \mathrm{~Hz}$ frequency range may be seen as being related to the finding of increased alpha $(9-12 \mathrm{~Hz})$ activity, primarily in posterior brain regions, during drowsiness or inattention. For example, Goldman et al. (2002) report that increases in alpha power when subjects close their eyes correlate with decreases in simultaneously recorded fMRI blood oxygen level-dependent signal in occipital lobe, superior temporal lobe, inferior frontal lobe, and cingulate cortex. In our task, decreases in alpha band oscillations during periods of heightened attention would produce a negative oscillatory SME in that frequency range. This does not, however, account for the negative SMEs found in the beta $(13-28 \mathrm{~Hz})$ frequency range. Although the negative alpha and beta SMEs are highly correlated (discrete correlation Yule's $Q=0.91 \pm 0.17$ ), the beta SMEs may reflect neurocognitive processes other than resting alpha, but until we have more detailed information concerning other behavioral correlates of oscillatory activity in the $13-28 \mathrm{~Hz}$ range, the present findings are best characterized phenomenologically.

Although we found a positive theta SME that lateralized to right frontal regions, fMRI studies typically find preferential activation of left prefrontal regions during episodic encoding (compared with retrieval) (Habib et al., 2003). It should be noted that we did not find significant lateralization of the positive gamma SME and that the negative SMEs in the alpha and beta frequency bands were slightly more prevalent in left compared with right prefrontal cortex. Given that our electrode positions were determined by clinical criteria and that we had relatively small numbers of subjects contributing electrodes in a given region, it is premature to interpret our findings of lateralization in the SME as being either consistent or inconsistent with the positron emission tomography and fMRI literature.

By examining intracranially recorded EEGs while subjects studied lists of words, we showed that increased oscillatory power during encoding predicts subsequent recall. Furthermore, this 
oscillatory subsequent memory effect localized to the $4-8 \mathrm{~Hz}$ (theta) and 28-64 Hz (gamma) frequency bands, with each effect exhibiting a unique brain topography. The increase in gamma activity at widespread cortical sites during successful encoding supports an emerging view of gamma as reflecting top-down attentional processes (Tiitinen et al., 1993; Debener et al., 2003). The increase in temporofrontal theta activity during successful encoding is consistent with physiological evidence concerning the role of theta in gating long-term potentiation. As such, this finding may help to bridge our understanding of the role of theta in synaptic plasticity with its role in human memory function.

\section{References}

Brewer JB, Zhao Z, Desmond JE, Glover GH, Gabrieli JD (1998) Making memories: brain activity that predicts how well visual experience will be remembered. Science 281:1185-1187.

Caplan JB, Madsen JR, Raghavachari S, Kahana MJ (2001) Distinct patterns of brain oscillations underlie two basic parameters of human maze learning. J Neurophysiol 86:368-380.

Caplan JB, Madsen JR, Schulze-Bonhage A, Aschenbrenner-Scheibe R, Newman EL, Kahana MJ (2003) Human theta oscillations related to sensorimotor integration and spatial learning. J Neurosci 23:4726-4736.

de Araujo DB, Baffa O, Wakai RT (2002) Theta oscillations and human navigation: a magnetoencephalography study. J Cognit Neurosci $14: 70-78$.

Debener S, Herrmann CS, Kranczioch C, Gembris D, Engel AK (2003) Topdown attentional processing enhances auditory evoked gamma band activity. NeuroReport 14:683-686.

De Gennaro L, Ferrara M, Ferlazzo F, Bertini M (2000) Slow eye movements and EEG power spectra during wake-sleep transition. Clin Neurophysiol 111:2107-2115.

Efron B (1979) Bootstrap methods: another look at the jackknife. Ann Stat $7: 1-26$.

Fell J, Klaver P, Lehnertz K, Grunwald T, Schaller C, Elger CE, Fernandez G (2001) Human memory formation is accompanied by rhinalhippocampal coupling and decoupling. Nat Neurosci 4:1259-1264.

Fell J, Klaver P, Elfadil H, Schaller C, Elger CE, Fernandez G (2003) Rhinalhippocampal theta coherence during declarative memory formation: interaction with gamma synchronization? Eur J Neurosci 17:1082-1088.

Fernandez G, Effern A, Grunwald T, Pezer N, Lehnertz K, Dumpelmann M, Van Roost D, Elger CE (1999) Real-time tracking of memory formation in the human rhinal cortex and hippocampus. Science 285:1582-1585.

Gevins A, Smith ME, McEvoy L, Yu D (1997) High-resolution EEG mapping of cortical activation related to working memory: effects of task difficulty, type of processing, and practice. Cereb Cortex 7:374-385.

Givens B (1996) Stimulus-evoked resetting of the dentate theta rhythm: relation to working memory. NeuroReport 8:159-163.

Givens BS, Olton DS (1990) Cholinergic and GABAergic modulation of medial septal area: effect on working memory. Behav Neurosci 104:849-855.

Goldman RI, Stern JM, Engel Jr J, Cohen MS (2002) Simultaneous EEG and fMRI of the alpha rhythm. NeuroReport 13:2487-2492.

Graimann B, Huggins JE, Levine SP, Pfurtscheller G (2002) Visualization of significant ERD/ERS patterns in multichannel EEG and ECoG data. Clin Neurophysiol 113:43-47.

Habib R, Nyberg L, Tulving E (2003) Hemispheric asymmetries of memory: the hera model revisited. Trends Cogn Sci 7:241-245.

Hasselmo ME, Bodelon C, Wyble BP (2002) A proposed function for hippocampal theta rhythm: separate phases of encoding and retrieval enhance reversal of prior learning. Neural Comput 14:793-817.

Hölscher C, Anwyl R, Rowan MJ (1997) Stimulation on the positive phase of hippocampal theta rhythm induces long-term potentiation that can be depotentiated by stimulation on the negative phase in area CAl in vivo. J Neurosci 17:6470-6477.

Howard M, Rizzuto D, Caplan J, Madsen J, Lisman J, Aschenbrenner-Scheibe R, Schultze-Bonhage A, Kahana MJ (2003) Gamma oscillations increase with working memory load in humans. Cereb Cortex 13:1369-1374.

Howard MW, Kahana MJ (1999) Contextual variability and serial position effects in free recall. J Exp Psychol Learn Mem Cogn 25:923-941.
Huerta PT, Lisman JE (1993) Heightened synaptic plasticity of hippocampal CA1 neurons during a cholinergically induced rhythmic state. Nature 364:723-725.

Jensen O, Lisman JE (1998) An oscillatory short-term memorybuffer model can account for data on the Sternberg task. J Neurosci 18:10688-10699.

Kahana MJ, Sekuler R, Caplan JB, Kirschen M, Madsen JR (1999) Human theta oscillations exhibit task dependence during virtual maze navigation. Nature 399:781-784.

Kahana MJ, Seelig D, Madsen JR (2001) Theta returns. Curr Opin Neurobiol 11:739-744.

Kirchhoff BA, Wagner AD, Maril A, Stern CE (2000) Prefrontal-temporal circuitry for episodic encoding and subsequent memory. J Neurosci 20:6173-6180.

Klimesch W (1999) EEG alpha and theta oscillations reflect cognitive and memory performance: a review and analysis. Brain Res Brain Res Rev 29:169-195.

Klimesch W, Dopplemayr M, Russegger H, Pachinger T (1996) Theta band power in the human scalp EEG and the encoding of new information. NeuroReport 7:1235-1240.

Klimesch W, Doppelmayr M, Schimke H, Ripper B (1997) Theta synchronization and alpha desynchronization in a memory task. Psychophysiology 34:169-76.

Krause CM, Sillanmäki L, Koivisto M, Saarela C, Häggqvist A, Laine M, Hämäläinen H (2000) The effects of memory load on event-related EEG desynchronization and synchronization. Clin Neurophysiol 111:2071-2078.

Lachaux JP, Rodriguez E, Martinerie J, Varela FJ (1999) Measuring phase synchrony in brain signals. Hum Brain Mapp 8:194-208.

Leutgeb S, Mizumori SJ (1999) Excitotoxic septal lesions result in spatial memory deficits and altered flexibility of hippocampal single-unit representations. J Neurosci 19:6661-6672.

Lisman JE, Idiart MA (1995) Storage of $7 \pm 2$ short-term memories in oscillatory subcycles. Science 267:1512-1515.

Paller KA, Wagner AD (2002) Observing the transformation of experience into memory. Trends Cogn Sci 6:93-102.

Pavlides C, Greenstein YJ, Grudman M, Winson J (1988) Long-term potentiation in the dentate gyrus is induced preferentially on the positive phase of theta-rhythm. Brain Res 439:383-387.

Raghavachari S, Rizzuto DS, Caplan JB, Kirschen MP, Bourgeois B, Madsen JR, Kahana MJ, Lisman JE (2001) Gating of human theta oscillations by a working memory task. J Neurosci 21:3175-3183.

Rizzuto DS, Madsen JR, Bromfield E, Schulze-Bonhage A, Seelig D, Aschenbrenner-Scheibe R, Kahana MJ (2003) Reset of human neocortical oscillations during a working memory task. Proc Natl Acad Sci USA 100:7931-7936.

Rodriguez E, George N, Lachaux JP, Martinerie J, Renault B, Varela FJ (1999) Perception's shadow: long-distance synchronization of human brain activity. Nature 397:430-433.

Rugg MD, Allan K (2000) Event-related potential studies of memory. In: The oxford handbook of memory (Tulving E, Craik FIM, eds), pp 521537. Oxford: Oxford UP.

Strange BA, Otten LJ, Josephs O, Rugg MD, Dolan RJ (2002) Dissociable human perirhinal, hippocampal, and parahippocampal roles during verbal encoding. J Neurosci 22:523-528.

Tallon-Baudry C, Bertrand O, Peronnet F, Pernier J (1998) Induced gamma-band activity during the delay of a visual short-term memory task in humans. J Neurosci 18:4244-4254.

Tesche CD, Karhu J (2000) Theta oscillations index human hippocampal activation during a working memory task. Proc Natl Acad Sci USA 97:919-924.

Tiitinen H, Sinkkonen J, Reinikainen K, Alho K, Lavikainen J, Naatanen R (1993) Selective attention enhances the auditory $40-\mathrm{Hz}$ transient response in humans. Nature 364:59-60.

Wagner AD, Schacter DL, Rotte M, Koutstaal W, Maril A, Dale AM, Rosen BR, Buckner RL (1998) Building memories: remembering and forgetting of verbal experiences as predicted by brain activity. Science 281:1188-1191.

Weiss S, Rappelsberger P (2000) Long-range EEG synchronization during word encoding correlates with successful memory performance. Cognit Brain Res 9:299-312. 